\title{
Stochastic Sensing of Polynucleotides Using Patterned Nanopores
}

\author{
Jack A. Cohen, ${ }^{1, *}$ Abhishek Chaudhuri, ${ }^{1,2, \dagger}$ and Ramin Golestanian ${ }^{1, \$}$ \\ ${ }^{1}$ Rudolf Peierls Centre for Theoretical Physics, University of Oxford, Oxford OX1 3NP, United Kingdom \\ ${ }^{2}$ Department of Biomedical Science, University of Sheffield, Sheffield S10 2TN, United Kingdom
}

(Received 21 December 2011; published 5 April 2012)

\begin{abstract}
The effect of the microscopic structure of a pore on polymer translocation is studied using Langevin dynamics simulation, and the consequence of introducing patterned stickiness inside the pore is investigated. It is found that the translocation process is extremely sensitive to the detailed structure of such patterns with faster than exponential dependence of translocation times on the stickiness of the pore. The stochastic nature of the translocation process leads to discernible differences between how polymers with different sequences go through specifically patterned pores. This notion is utilized to propose a stochastic sensing protocol for polynucleotides, and it is demonstrated that the method, which would be significantly faster than the existing methods, could be made arbitrarily robust.
\end{abstract}

DOI: 10.1103/PhysRevX.2.021002

Subject Areas: Biological Physics, Statistical Physics, Soft Matter

\section{INTRODUCTION}

The quest for efficient sequencing of single-stranded DNA using synthetic nanopores has recently led to the development of a plethora of novel theoretical and experimental design ideas that use a variety of different approaches [1-7]. Experiments have demonstrated that the current blockade readout from single-stranded DNA (ssDNA) and RNA molecules that are electrophoretically driven through biological and synthetic nanopores could in principle reflect a signature of the underlying sequence [8-14]. It is now possible to design solid-state nanopores $[15,16]$ with tailored surface properties that could regulate DNA-pore surface interaction [17-19] and also reduce noise [20,21]. A number of recent experiments have been successful in discriminating between polynucleotides [22] and identifying single nucleotides [23-25]. However, more remains to be done to resolve issues involving stability, sensitivity, and resolution, before they can be integrated into fast and efficient devices for sequencing purposes $[26,27]$.

Theoretical studies of polymer translocation through nanopores [28-51] have revealed that the process is intrinsically stochastic and features a rather wide distribution for the translocation time. The inherent noise acts as an overwhelming source of error for the sequence detection strategies that are based on deterministic patterns in the translocation time readout. Sequencing methods based on current-blockade measurements require that the process is sufficiently slowed down such that time-averaging

\footnotetext{
*j.cohen@physics.ox.ac.uk

a.chaudhuri1@physics.ox.ac.uk

ramin.golestanian@physics.ox.ac.uk
}

Published by the American Physical Society under the terms of the Creative Commons Attribution 3.0 License. Further distribution of this work must maintain attribution to the author(s) and the published article's title, journal citation, and DOI. eliminates the noise $[8,22]$. In other words, achieving robustness in sequencing using deterministic strategies has intrinsic limitations, and might require significant compromise in translocation speed [3].

Here, we propose a strategy to control the translocation time of ssDNA and its statistics by using pores that have patterned surface energetics. We then address the question of whether it is possible to engineer distinct stochastic features for the translocation of single-stranded heteropolymers with any given sequence through different pores, such that the statistical readout from combined translocation events of a particular sample through a collection of different pores could quickly and accurately reveal its sequence by synergistic exclusion of unlikely sequences. We study the translocation of polymers, driven from the cis (entrance) to the trans (exit) side of a narrow pore by a uniform external field, $F$ (Fig. 1; see Sec. II). We vary the stickiness of the pore (characterized by the attractive strength, $\left.\epsilon_{\mathrm{pm}}\right)$ along its length and consider three different examples [Figs. 1(a)-1(c)]. A uniformly attractive pore, Pore $\alpha$, serves as the control case. Pore $\beta$ is structured to have an attractive entrance and exit separated by a shortranged repulsive core, while Pore $\gamma$ is designed to have an attractive entrance and a repulsive exit. These apparently minor changes in the pore patterning turn out to have significant effects on the translocation times.

\section{METHODS}

Homopolymer model. We model the polymer as a selfavoiding chain by using beads and springs (Fig. 1). The beads represent monomer groups of the polymer with an excluded volume interaction between a pair of monomers set by a truncated repulsive Lennard-Jones (rLJ) potential of the form

$$
U_{\mathrm{mm}}^{\mathrm{LJ}}(r)= \begin{cases}4 \epsilon\left[\left(\frac{\sigma}{r}\right)^{12}-\left(\frac{\sigma}{r}\right)^{6}\right]+\epsilon & : r \leq r_{\text {min }} \\ 0 & : r>r_{\text {min }}\end{cases}
$$




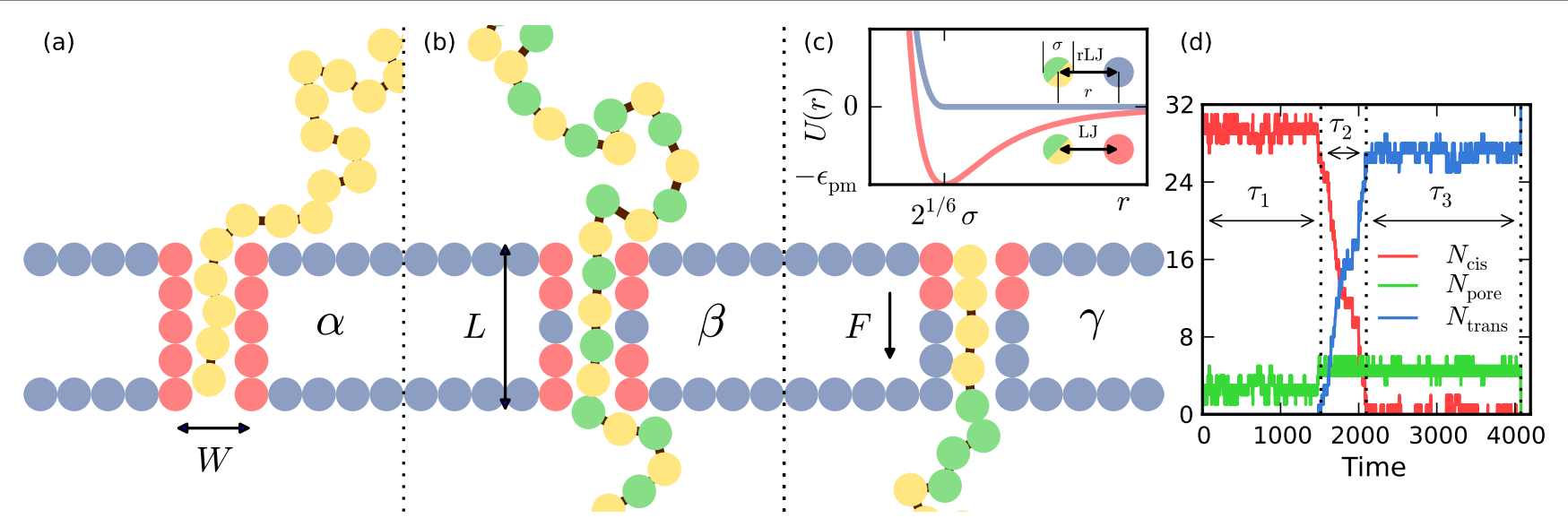

FIG. 1. The schematics of the polymer and nanopore models. Simulation snapshots showing the translocation of a homopolymer and heteropolymers with different block lengths across the three patterned pores at various stages of the translocation process, namely, filling, transfer, and escape. (a) A homopolymer (yellow) translocating from the cis to the trans side of Pore $\alpha$. The interaction of the pore monomers (red) with the polymer has an attractive well whereas the monomers that make up the walls of the pore (blue) have an excluded volume interaction with the polymer. The pore width is fixed and there is a constant force driving the polymer that acts inside the pore. (b) A heteropolymer (poly $(\mathrm{dAdC})_{16}$ ) of block length $M=2$ with alternating bases A (yellow) and C (green) translocating across Pore $\beta$. Pore $\beta$ consists of two sticky monomers on either end of the pore that are separated by a wall monomer. The bases $\mathrm{A}$ and $\mathrm{C}$ have different interactions with the sticky monomers. (c) A heteropolymer (poly $\left.\left(\mathrm{dA}_{4} \mathrm{dC}_{4}\right)_{4}\right)$ of block length $M=8$ translocating across Pore $\gamma$, which has two sticky monomers on the cis side and three repulsive monomers on the trans side that result in an attractive entrance and a repulsive exit. (Inset) Shows the pore-polymer potentials. (d) A trace of the monomer count at the trans end, middle, and cis end of the pore as functions of time for a homopolymer translocating through Pore $\gamma$, with $\epsilon_{\mathrm{pm}}=3$ and $F=0.5 . \tau_{1}, \tau_{2}$, and $\tau_{3}$ change dramatically when pore patterning is introduced.

where $\epsilon$ is the potential depth and $\sigma$ is the monomer diameter. The cutoff distance, $r_{\min }=2^{1 / 6} \sigma$, is set at the potential minimum. The monomers are bonded by a finite extension nonlinear elastic (FENE) potential of the form

$$
U_{\mathrm{ch}}^{\mathrm{FENE}}(r)=-\frac{1}{2} k R^{2} \ln \left(1-\frac{r^{2}}{R^{2}}\right)
$$

where $k=7 \epsilon / \sigma^{2}$ and $R=2 \sigma$ are the spring constant and bond length, respectively. FENE potentials are convenient as the bond length sets the maximum allowed separation between monomer groups. We use polymers of length $N=32$ and $N=64$ in our simulations.

Heteropolymer model. We model the heteropolymers similarly using beads and springs (Fig. 1) with the polymer beads representing the bases $\mathrm{A}$ and $\mathrm{C}$ arranged in symmetric blocks $A_{n} C_{n}$. With a DNA of length $N=32$, the minimum value of $n=1$ is for poly $(\mathrm{dAdC})_{16}$ and the maximum value of $n=N / 2$ for poly $\left(\mathrm{dA}_{16} \mathrm{dC}_{16}\right)$. The bases $\mathrm{A}$ and $\mathrm{C}$ are only distinguished by their relative interactions with the pore.

Pore model. The pore and wall are constructed from stationary monomers separated by a distance of $\sigma$ from each other. The pore is made up of two rows of monomers symmetric about the coordinate system with a length $L=$ $5 \sigma$ and width $W=2.25 \sigma$. The pore width is chosen to allow only single-file translocation of the polymer and avoid hairpin configurations. The polymer translocates from the cis (entrance) end to the trans (exit) end of the pore (Fig. 1).
Polymer-pore interaction. The interaction of the pore with the polymer is tuned such that the interaction varies along the length of the pore. This interaction could either be the short-range repulsive form described above or the standard LJ form:

$$
U_{\mathrm{pm}}^{\mathrm{LJ}}(r)= \begin{cases}4 \epsilon_{\mathrm{pm}}\left[\left(\frac{\sigma}{r}\right)^{12}-\left(\frac{\sigma}{r}\right)^{6}\right] & : r \leq r_{c} \\ 0 & : r>r_{c}\end{cases}
$$

with $\epsilon_{\mathrm{pm}}$ denoting the potential depth and $r_{c}=2.5 \sigma$ denoting the cutoff distance. We choose three different pore patterns : (1) Pore $\alpha$ is an attractive pore with all the monomers of the pore interacting with the polymer by the LJ potential. (2) Pore $\beta$ has an attractive entrance and exit with the first two monomers and the last two monomers of the pore interacting with the polymer by the LJ potential and the middle monomer being repulsive. (3) Pore $\gamma$ has an attractive entrance (first two monomers attractive) and a repulsive exit (last three monomers repulsive). Note that in all the three cases the pore entrance is chosen to be attractive to successfully initiate translocation. The stickiness of the pore $\left(\epsilon_{\mathrm{pm}}\right)$ is varied for homopolymer translocation. For the translocation of the heteropolymer the stickiness differs for base $\mathrm{A}\left(\epsilon_{\mathrm{pA}}\right)$ and base $\mathrm{C}\left(\epsilon_{\mathrm{pC}}\right)$. We fix these values to $\epsilon_{\mathrm{pA}}=3 \epsilon$ and $\epsilon_{\mathrm{pC}}=\epsilon$ respectively. The polymer interacts with the wall $\left(U_{\mathrm{mw}}^{\mathrm{LJ}}\right)$ with the same rLJ potential as used for the intramonomer excluded volume interaction. In addition the polymer experiences a driving force, $\mathbf{F}_{\mathrm{e}}=F \hat{\mathbf{x}}$ directed along the pore 
axis with magnitude $F$, which mimics the electrophoretic driving of biopolymers through nanopores.

Polymer injection. In our simulation we are not concerned with injection of the polymer into the pore, but only with the dynamics of the polymer during translocation. We initially place the first bead of the polymer chain at the entrance of the pore and allow the remaining beads to fluctuate. Once the polymer relaxes to its equilibrium configuration, the bead is released and the translocation of the polymer across the pore is monitored. The translocation time is defined as the time that elapses between the entrance of the first bead of the polymer and the exit of the last bead. All failed translocation events are discarded.

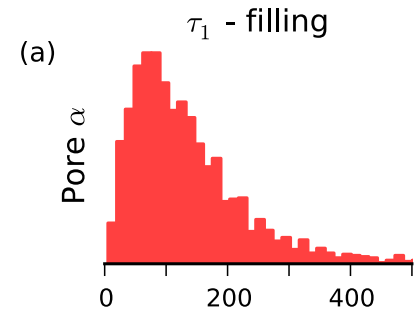

(b)
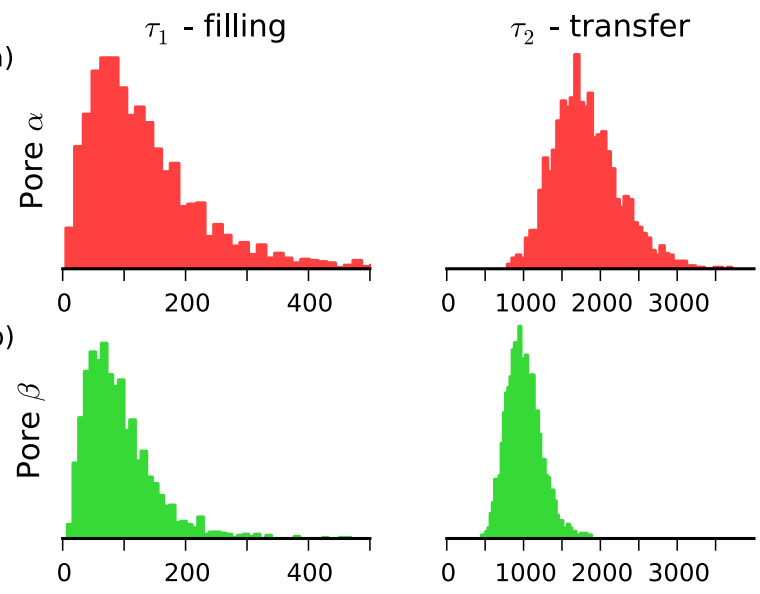

(c)
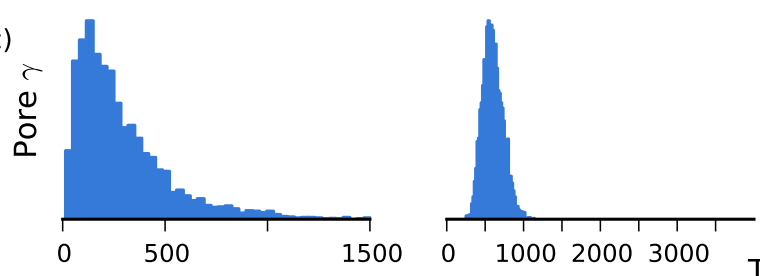

(d) $\tau_{1}$

(e) $\tau_{2}$

(f) $\tau_{3}$
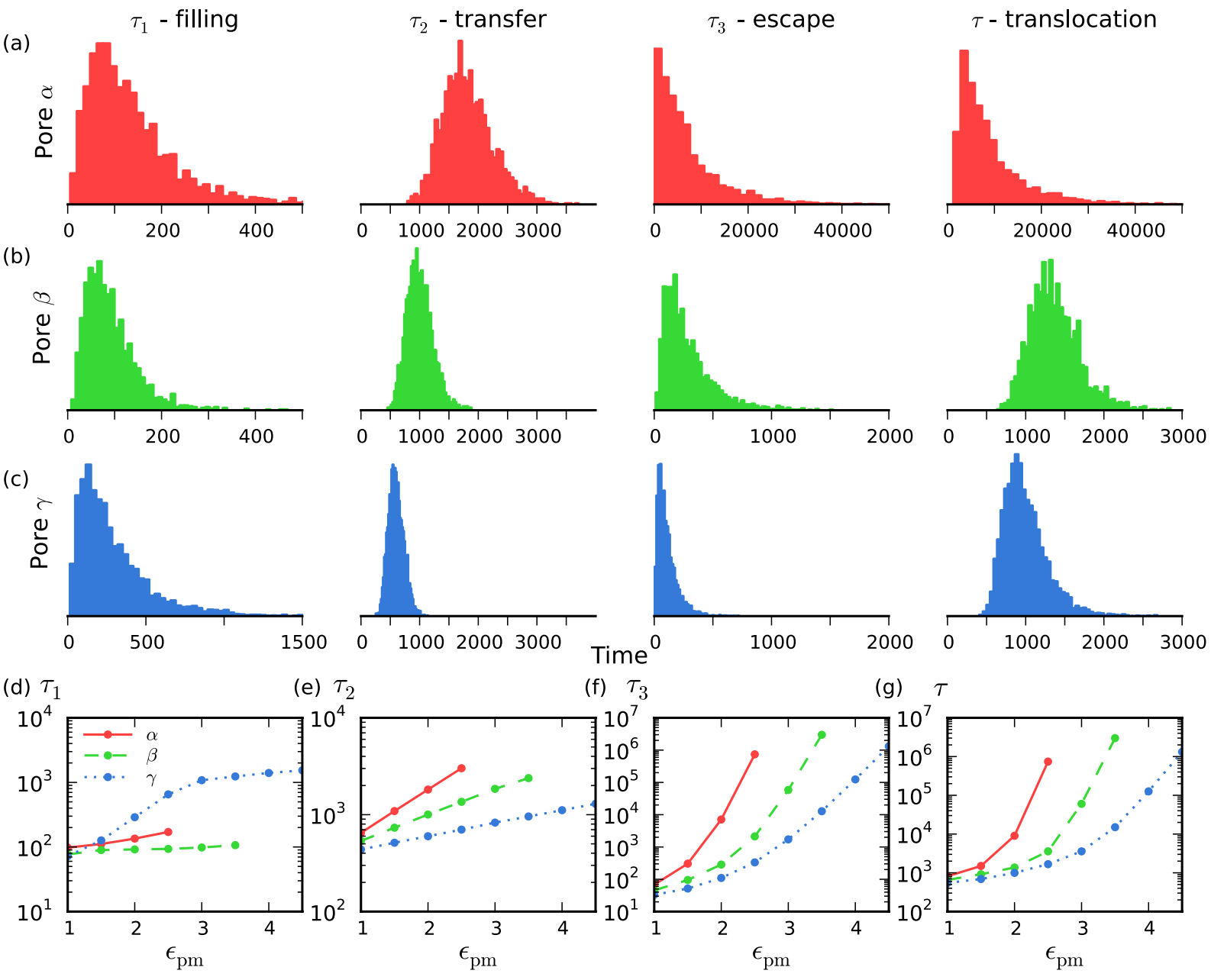

FIG. 2. Translocation time statistics for homopolymers. (a), (b), and (c) Comparison of translocation time distributions for the three patterned pores, for $F=0.5$ and $\epsilon_{\mathrm{pm}}=2.0$. The filling, transfer, and escape distributions are similar across the three pores, but have distinctly different scales (e.g., average and variance), such that the overall translocation time distribution for the three pores is discernibly different. (d), (e), and (f) Comparison of average filling, transfer, and escape times for the three different pores for $F=0.5$ as a function of $\epsilon_{\mathrm{pm}}$. While the filling time shows only a moderate dependence on the stickiness, and the transfer time exhibits an exponential dependence on $\epsilon_{\mathrm{pm}}$, the dependence of the escape time is even faster than exponential. (g) The total translocation time for the three pores as a function of $\epsilon_{\mathrm{pm}}$ indicates that for small forces, the escape time dominates the translocation process. The orders of magnitude differences in the translocation times between different pores show the extraordinary sensitivity of the translocation dynamics on pore patterning. 


\section{t: $500.0 \quad$ Golestanian Group $\quad \epsilon_{\mathrm{pm}}=2.0 \quad F=0.5$}
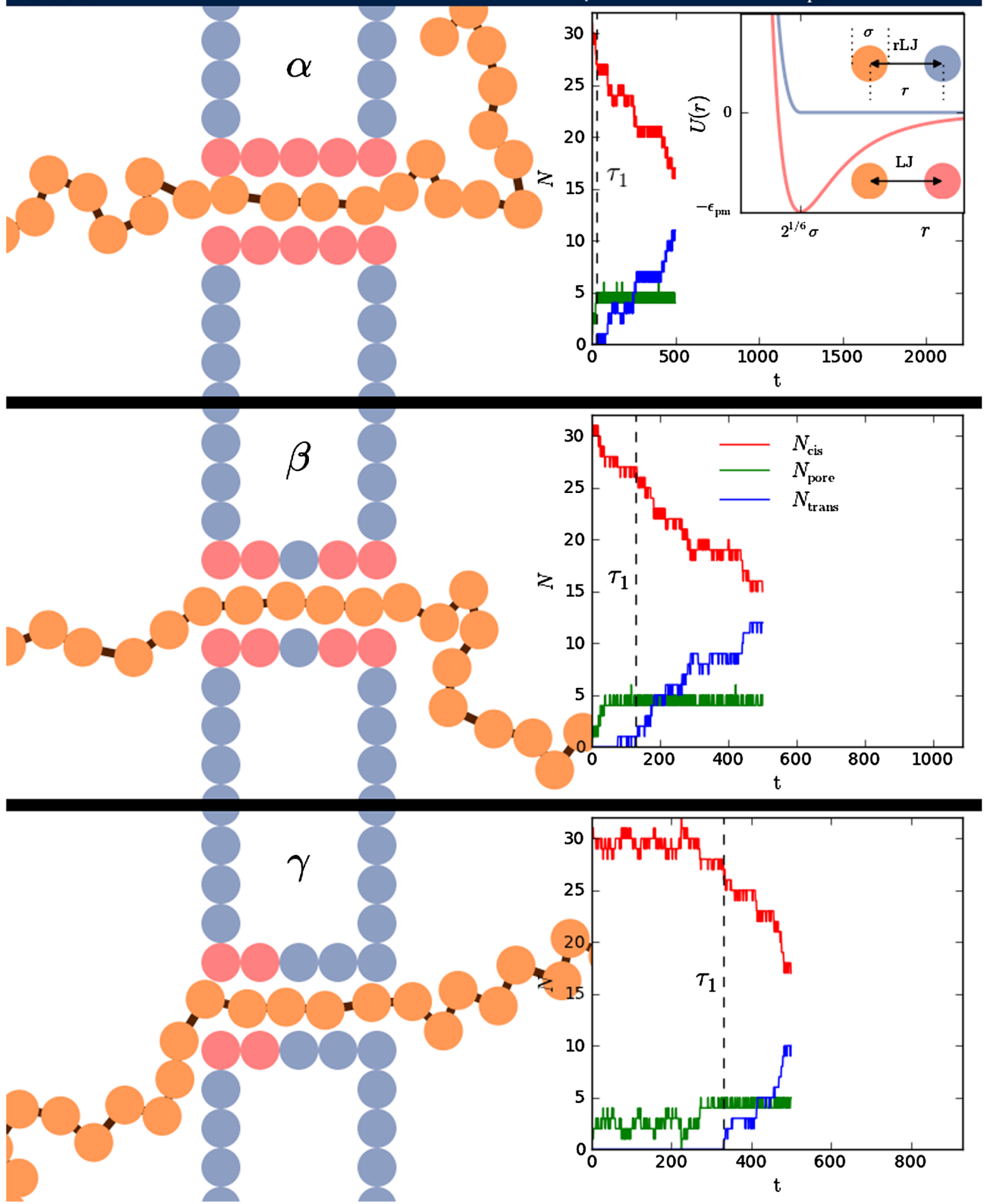

VIDEO 1. Homopolymer translocating through Pores $\alpha, \beta$, and $\gamma$. A homopolymer of length $N=32$ is shown translocating through the three pore patterns considered in this study. The upper panel shows Pore $\alpha$, which is attractive throughout, the middle panel shows Pore $\beta$, which has a short-range repulsive core, and the lower panel shows Pore $\gamma$, which has an attractive entrance. On the right, the number of monomers is counted in the cis (red), pore (green), and trans (blue) regions as a function of time for each pore. The filling, $\tau_{1}$, transfer, $\tau_{2}$, and escape time, $\tau_{3}$, are marked when the event occurs. The runs selected have a translocation time near that of the mean translocation time. The parameters, as explained in the paper, are $W=2.25, \epsilon_{\mathrm{pm}}=2.0$, and $F=0.5$. 
monomer, $\zeta$ is the friction coefficient, $\mathbf{v}_{i}$ is the monomer velocity, and $\eta_{i}$ is the random force with $\left\langle\eta(t) \cdot \eta\left(t_{0}\right)\right\rangle=$ $4 k_{\mathrm{B}} T \zeta \delta_{i j} \delta\left(t-t_{0}\right), T$ being the temperature. A time step of $\Delta t=0.01$ is used in all simulation runs.

Reduced units. The units of energy, length, and mass are set by $\epsilon, \sigma$, and $m$, respectively. These set the scale for the time as $\left(m \sigma^{2} / \epsilon\right)^{1 / 2}$. Following Luo et al. [34], we assume that the size of each bead in our coarse-grained polymer model corresponds to the Kuhn length of a single-stranded DNA, which is approximately three nucleotide bases. This sets the bead size, $\sigma \approx 1.5 \mathrm{~nm}$, the mass of the bead, $m \approx$ $936 \mathrm{amu}$ (given that the mass of a base in DNA is $\approx$ $312 \mathrm{amu}$ ) and the charge of a bead, $q \approx 0.3$ e (each base having a charge of 0.1 e effectively [53]). We set $\zeta=0.7$ and $k_{B} T=1.2$ to allow comparison with known results. Therefore, the interaction strength at $T=295 \mathrm{~K}$ is given by $\epsilon=k_{B} T / 1.2 \approx 3.4 \times 10^{-21} \mathrm{~J}$. This gives the time scale of $\left(m \sigma^{2} / \epsilon\right)^{1 / 2} \approx 30 \mathrm{ps}$ and a force scale of $\epsilon / \sigma \approx$ $2.3 \mathrm{pN}$. Therefore an external driving force in the range $0.5-1.0$ corresponds to a voltage range $V=F L / q \approx$ 190-380 mV across the pores. Note, however, that higher values of up to 0.5 e for the effective base charge have also been reported in the literature [54], which suggest that the appropriate voltage range could be lower than the abovementioned values. As a rough indication of how much the patterning could affect translocation speed, we note the example of a homopolymer with $\epsilon_{\mathrm{pm}}=2.5$ [see Fig. 2(g)], which yields the translocation time of $100 \mu$ s for Pore $\alpha$ and $0.1 \mu$ s for Pore $\gamma$. These are consistent with the typical [3] observed translocation rates of a single nucleotide/ $\mu \mathrm{s}$.

\section{TRANSLOCATION TIME DISTRIBUTIONS FOR HOMOPOLYMERS}

The translocation time $(\tau)$ is divided into (i) the initial filling time $\left(\tau_{1}\right)$ : the time taken by the first monomer of the polymer to reach the exit without returning to the pore; (ii) the transfer time $\left(\tau_{2}\right)$ : the time taken from the exit of the first monomer into the trans side to the entry of the last monomer from the cis side; and (iii) the escape time $\left(\tau_{3}\right)$ : the time between entry of the last monomer in the pore and its escape to the trans side [see Figs. 1(a)-1(c)]. These definitions are better characterized by counting the number of monomers of the polymer on the cis side, $N_{\text {cis }}$, inside the pore, $N_{\text {pore }}$, and on the trans side, $N_{\text {trans }}$, as functions of time [Fig. 1(d)], with $N=N_{\text {cis }}+N_{\text {pore }}+N_{\text {trans }}$ (see Video 1).

For Pores $\alpha$ and $\beta$, the filling time, $\tau_{1}$, depends weakly on the stickiness of the pore [Fig. 2(d)]. In the presence of the weak driving force, Pore $\beta$ has a shallower potential well near the cis end, which reduces trapping time making filling easier (see Appendix B). The barrier encountered near the core is small enough to be overcome by the fluctuations of the polymer. With increasing $\epsilon_{\mathrm{pm}}$, the effect of trapping becomes more dominant and thus the difference in $\tau_{1}$ between pores $\alpha$ and $\beta$ increases.
Pore $\gamma$, which has a repulsive exit, takes a relatively longer time to fill. The large potential barrier beyond the cis side slows down the polymer increasingly more as the entrance becomes stickier (with increasing $\epsilon_{\mathrm{pm}}$ ). The distribution of filling times shows a relatively longer exponential tail for Pore $\gamma$ due to this potential barrier. In sharp contrast to the filling time, the transfer time $\tau_{2}$ has a much more regular behavior with increasing stickiness of the pores [Fig. 2(e)]. The transfer of the polymer over the length of the pore depends on the potential landscape inside the pore: Pore $\alpha$, which is attractive throughout, has the longest transfer time, while Pore $\gamma$, which is the least attractive pore, has the shortest $\tau_{2}$. Figure 2(e) shows that the transfer time depends exponentially on $\epsilon_{\mathrm{pm}}$, and the difference in scales across the three pores is consistent with the number of attractive beads inside each pore. The escape time, $\tau_{3}$, depends strongly on the pore interaction near the exit, and differs most dramatically across the three pores [Fig. 2(f)]. In this time interval, the polymer is already inside the pore and to escape the pore it needs to overcome the potential barrier near the exit. The dependence of the escape time on $\epsilon_{\mathrm{pm}}$ turns out to be faster than exponential, which suggests that seemingly insignificant changes in the stickiness pattern and strength of the wall of the pore could modify the translocation time by several orders of magnitude.

The average total translocation time for the homopolymer across all the pores is plotted in Fig. 2(g), which shows that for the relatively weak external force used here the translocation process is controlled by the escape mode [see Figs. 2(d)-2(g)]. The overall translocation time distributions for the three pores are also very different [Figs. 2(a)-2(c)], despite the fact that the general shape of the distributions for each mode of the translocation process was similar. The extreme sensitivity of the translocation dynamics of the homopolymer on the pore patterning and stickiness suggests that it might be possible to engineer pores such that heteropolymers of any given sequence will have distinct statistical features that could be used for stochastic sequence detection.

\section{HETEROPOLYMER SEQUENCE SENSING}

To examine the feasibility of this sequencing strategy, we replace the homopolymer with heteropolymers constructed in accordance with earlier experimental [12] and theoretical [34] studies of polynucleotide translocation through nanopores: those containing symmetric purinepyrimidine blocks of the form $A_{n} C_{n}$, with the block length $M=2 n$ (Fig. 1). We assign different values to the attractive interactions of the sticky beads in the pore with base $\mathrm{A}\left(\epsilon_{\mathrm{pA}}\right)$ and base $\mathrm{C}\left(\epsilon_{\mathrm{pC}}\right)$, with $\epsilon_{\mathrm{pA}}>\epsilon_{\mathrm{pC}}$.

The translocation time distributions for five different sequences are shown in Figs. 3(a) and 3(b) for Pores $\beta$ and $\gamma$. We find that the different modes of translocation across the two pores respond differently to variations 

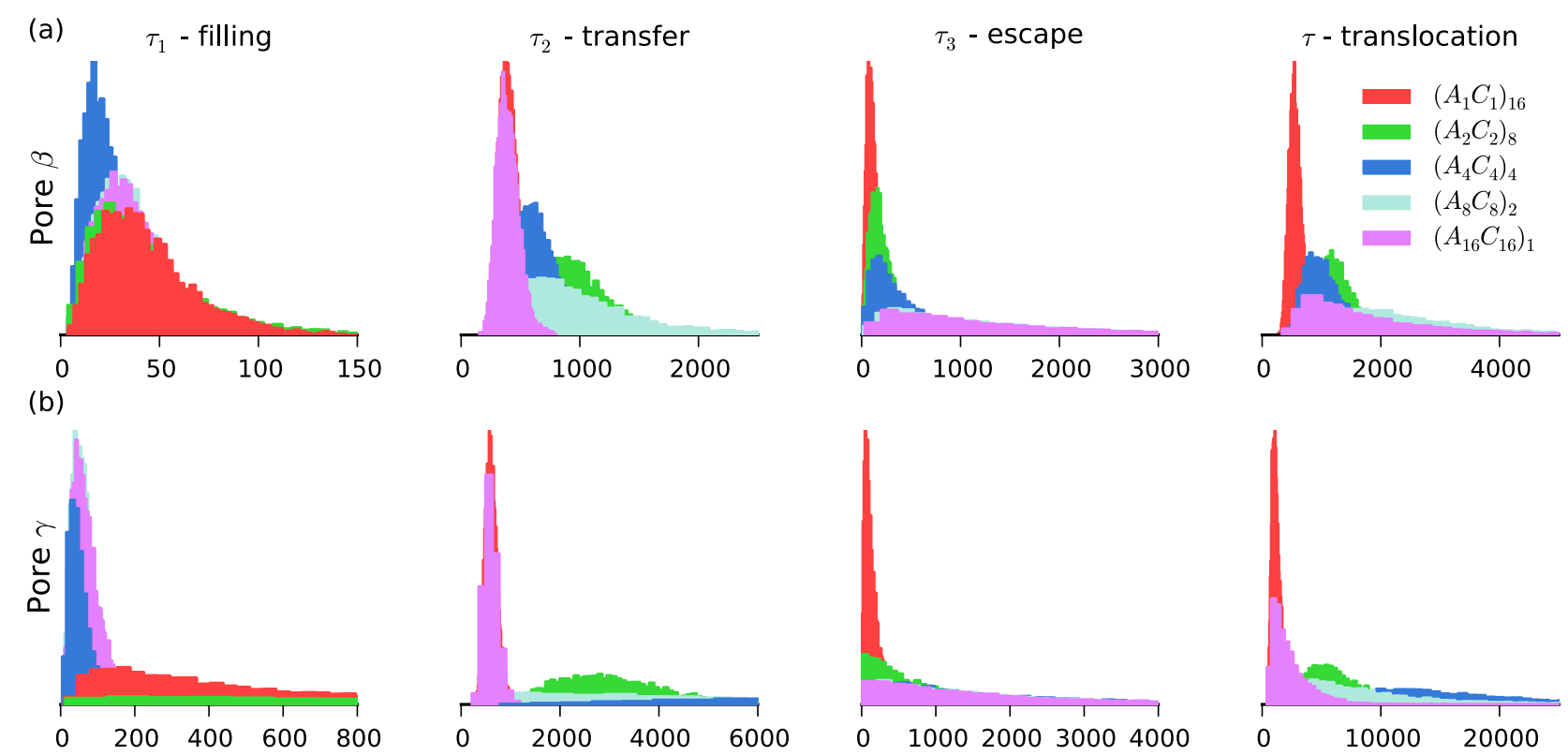

FIG. 3. Translocation time statistics for heteropolymers. Comparison of filling, transfer, escape, and translocation time distributions for Pores $\beta$ (a) and $\gamma$ (b) and five different sequences of the heteropolymer. The distributions correspond to $F=1.0$ for Pore $\beta$, and $F=0.5$ for Pore $\gamma$, respectively.

in the block length, such that the outcome for the total translocation time exhibits distinct features (see Videos 2 and 3). To simplify the picture, we summarize the distributions for each pore in a scatter plot by using only the two basic characteristics of mean and standard deviation [Figs. 4(a)-4(d)]. We observe a number of interesting features. For example, both mean time and standard deviation seem to roughly increase with block length for Pore $\beta$, whereas for Pore $\gamma$ mean time initially increases with block length, peaks at $n=4$, and goes back to smaller values for longer blocks. While Pore $\beta$ cannot easily distinguish between $\left(A_{4} C_{4}\right)_{4}$ and $\left(A_{2} C_{2}\right)_{8}$, Pore $\gamma$ can,

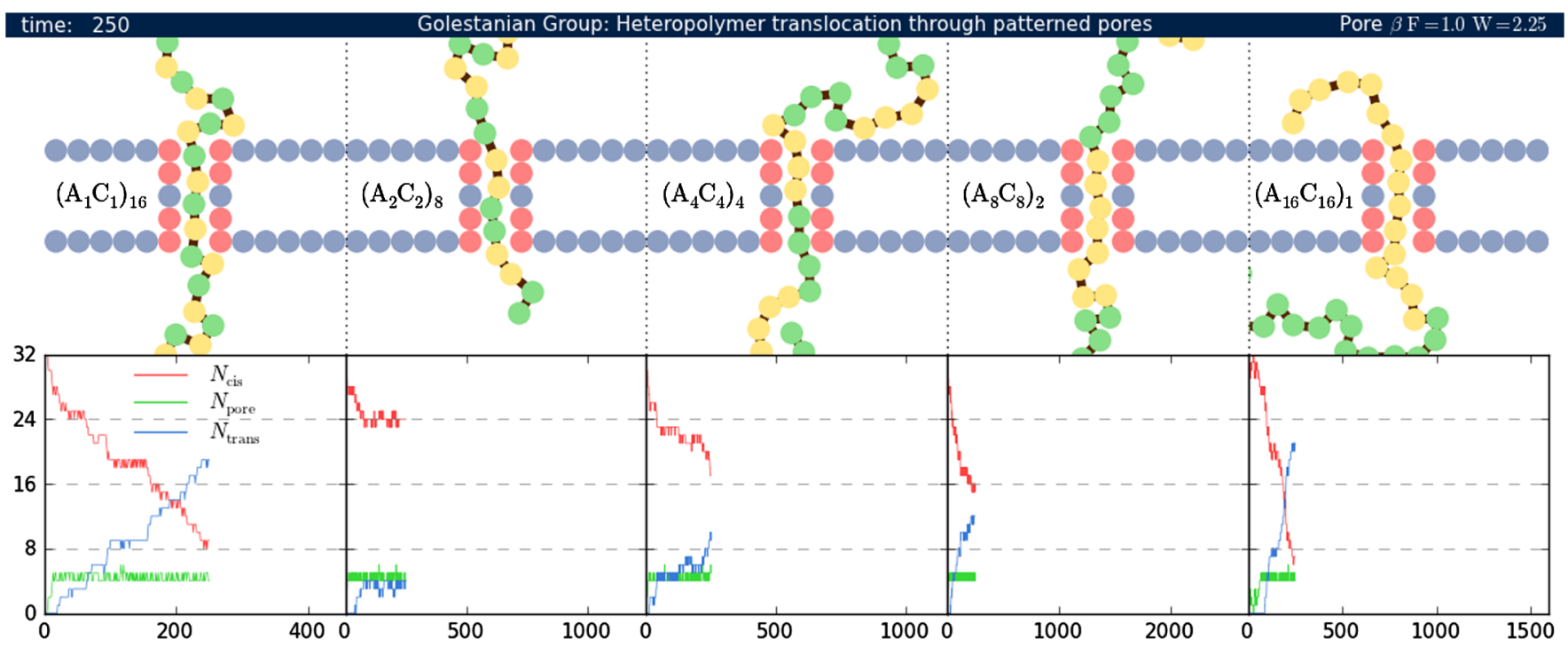

VIDEO 2. Heteropolymers translocating through Pore $\beta$. Heteropolymers of length $N=32$ with block lengths $1,2,4,8$ and 16 translocating through Pore $\beta$. Monomer type A (yellow) has an interaction energy three times larger than that of B (green). The top panel shows a view of the simulation near the pore region, which consists of attractive (red) and short-range repulsive (blue) patches. Pore $\beta$ is symmetric with a repulsive core. The total number of monomers in the cis (red), pore (green), and trans (blue) side are counted as a function of time in the lower plot. The runs selected have a translocation time near that of the mean of the distribution of translocation times for the particular structure. The parameters, as explained in the paper, are $W=2.25$ and $F=1.0$. 


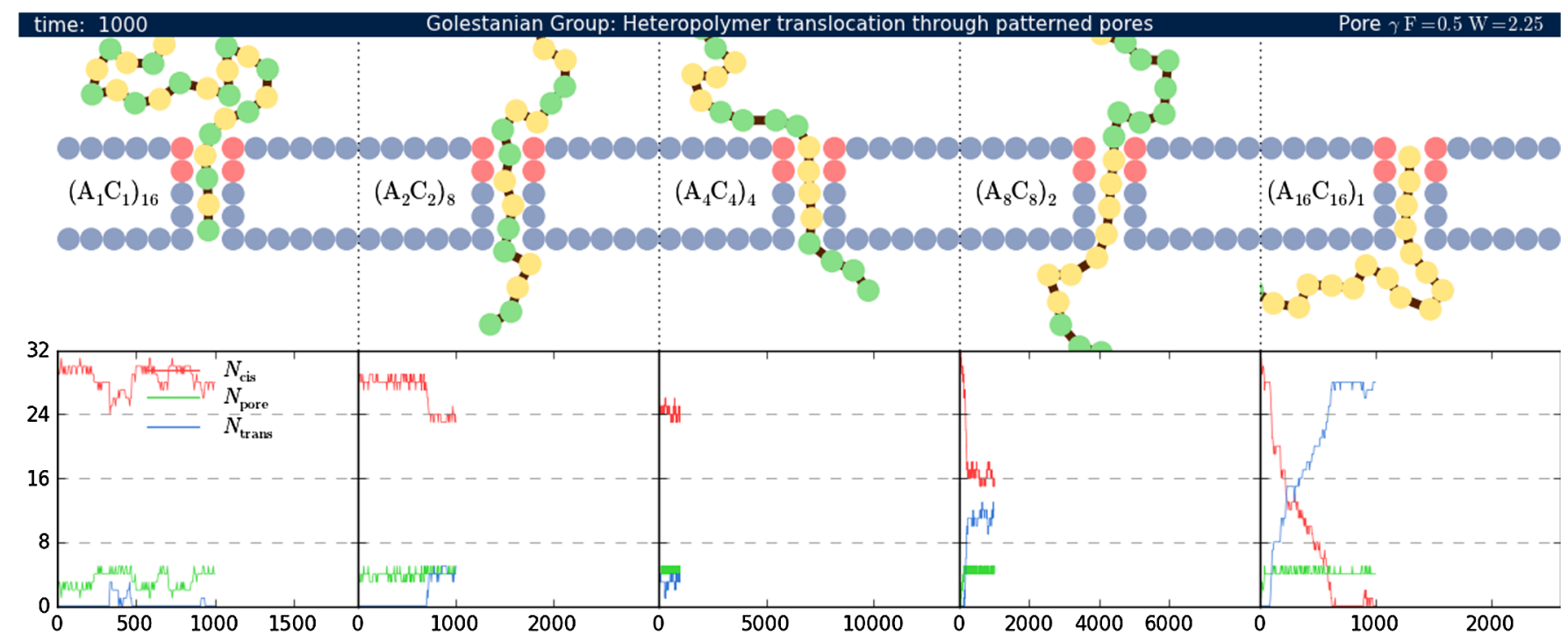

VIDEO 3. Heteropolymers of length $N=32$ with block lengths 1, 2, 4, 8 and 16 translocating through Pore $\alpha$. Monomer type A (yellow) has an interaction energy three times larger than that of B (green). The top panel shows a view of the simulation near the pore region, which consists of attractive (red) and short-range repulsive (blue) patches. Pore $\alpha$ is asymmetric with an attractive entrance. The total number of monomers in the cis (red), pore (green), and trans (blue) side are counted as a function of time in the lower plot. The runs selected have a translocation time near that of the mean translocation time for the particular structure. The parameters, as explained in the paper, are $W=2.25$ and $F=1.0$.

and the reverse is true for $\left(A_{4} C_{4}\right)_{4}$ and $\left(A_{8} C_{8}\right)_{2}$. We have also examined the effect of the orientation of the heteropolymer when it enters the pore, and considered polymers of total length $N=32$ and $N=64$ [Figs. 4(a)-4(d)]. The differences in the scatter immediately suggest that a combined translocation time measurement across the two pores and comparison with the statistics of the known sequences could help identify an unknown sequence to a high accuracy.

To demonstrate this idea and probe its statistical robustness as a sequencing strategy, we run a test on a model sequencing device that would be made up of multiple copies of Pores $\beta$ and $\gamma$ that are arranged in-series, such that the readout from translocation of a given polynucleotide with an unknown sequence through all of them can be independently recorded. We calculate the average and standard deviation of the translocation times through the Pores $\beta$ and $\gamma$, separately, using their corresponding multiple readouts. Using the difference between the measured means and standard deviations and the tabulated values for known sequences through each pore, we calculate the relative error for each sequence and minimize it for all sequences across both pores to find the closest match, which will be returned as the predicted sequence. The ratio of the number of successful sequence detection events and the total number of attempts, which is defined as the accuracy of the statistical sequence detection algorithm, turns out to be remarkably high [Fig. 4(e)]. For $N=32$ and fixed orientation of the polynucleotide for all pores, the accuracy starts off at $75 \%$ with just the minimum two copies of each pore and rises quickly to above $95 \%$ when there are ten copies of each pore.

Instead of just using the first two moments, we can choose to use the full translocation time distributions for the sequence detection, using the following method. If we make a measurement of the translocation time $(\tau)$ of a polymer with an unknown sequence through a given pore (Pore $\beta$, for example), then the probability of the time being part of a distribution of a known sequence (say $n$ ) is $\mathcal{P}_{n}^{\beta}(\tau)$, where $\mathcal{P}_{n}$ is the known probability distribution. After $m$ measurements, the likelihood of the translocation times being part of a given distribution can be defined as $\mathcal{L}_{n}=\Pi_{i=1}^{m} \mathcal{P}_{n}^{\beta}\left(\tau_{i}\right)$. The structure of the unknown heteropolymer is determined by finding the $n$ with the maximum likelihood, seq $\equiv \operatorname{seq}\left[\max \left\{\mathcal{L}_{n}\right\}\right]$. For multiple pores and fixed orientation of the polymer through all of them, the likelihood can be generalized to $\mathcal{L}_{n}^{\text {fo }}=$ $\Pi_{i=1}^{m} \mathcal{P}_{n}^{\beta}\left(\tau_{i}\right) \Pi_{i=1}^{m} \mathcal{P}_{n}^{\gamma}\left(\tau_{i}\right) \ldots$. Figure $4(\mathrm{e})$ shows the resulting accuracy plots as obtained using the full translocation time distributions, which exhibit a considerably faster convergence in the algorithm. The corresponding results are very similar for $N=64$ [Fig. 4(e)]. This shows that an inherently statistical DNA sequencing strategy could be designed to have an arbitrary accuracy.

During the sequence detection process the heteropolymer could enter the pore with either base A or base C entering first. Therefore it is imperative to consider orientation effects on the translocation time distributions, as seen in Figs. 4(a)-4(d), and hence on our sequencing strategy. Because of the possibility of orientation flips 

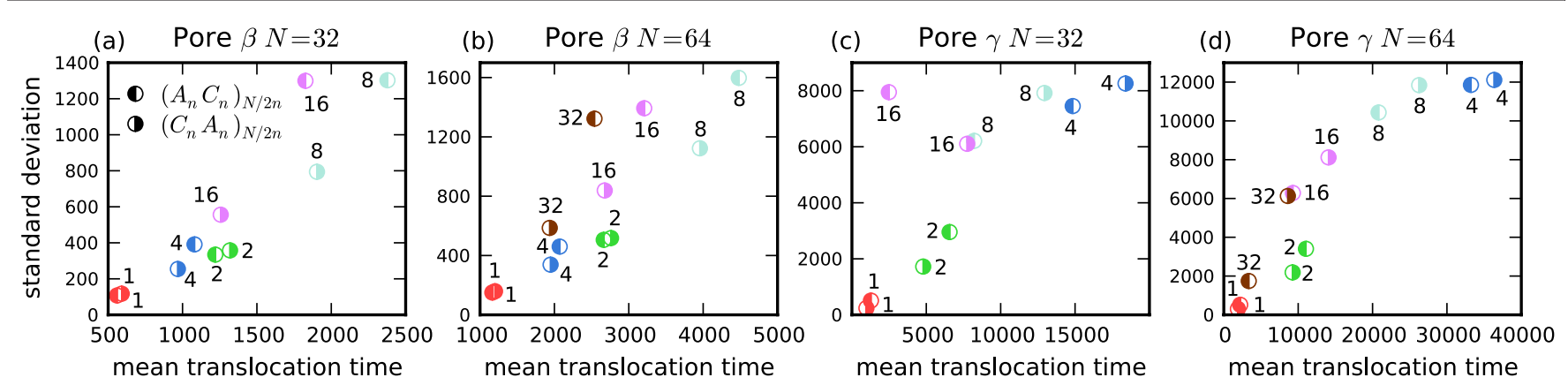

(e)

Fixed Orientation

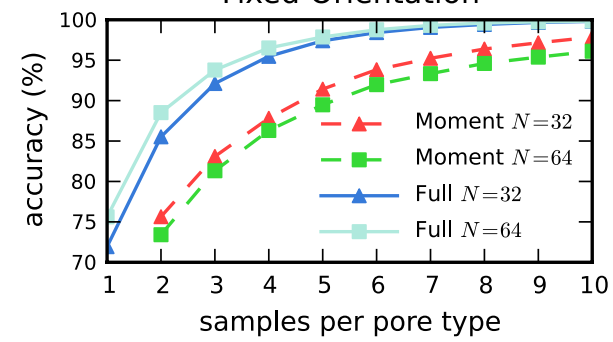

(f)

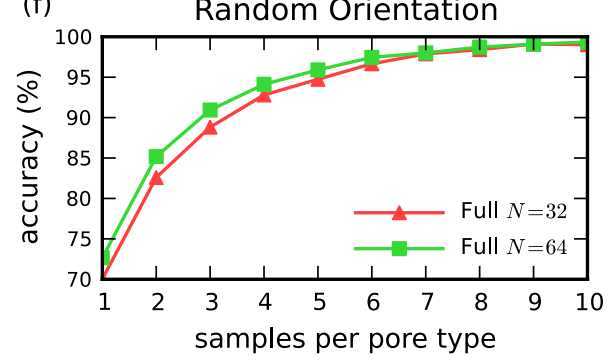

FIG. 4. Using translocation time statistics to detect polynucleotide sequences. (a), (b), (c), and (d) Scatter plots showing the distinctive mean and standard deviations of the different sequences and for different ends of the polynucleotide entering the pore. The mean translocation time, $\langle\tau\rangle$, and its standard deviation, $\sqrt{\left\langle\tau^{2}\right\rangle-\langle\tau\rangle^{2}}$, for the different sequences are calculated from the distributions. The scatter plots reveal the distinctive characteristics of the translocation events for the different sequences through each pore. The plots correspond to (a) Pore $\beta$ with $F=1.0$ and $N=32$; (b) Pore $\beta$ with $F=1.0$ and $N=64$; (c) Pore $\gamma$ with $F=0.5$ and $N=32$; and (d) Pore $\gamma$ with $F=0.5$ and $N=64$. (e) and (f) Accuracy of sequence detection using multiple joint translocation events through Pores $\beta$ and $\gamma$. The plots are constructed by recording a given number of translocation times through Pores $\beta$ and $\gamma$, and using a comparison with either the full distribution or the first two moments of the distribution shown in the scatter plots (a, b, c, and d). For the method that uses the moments, the sample average and standard deviation are calculated and used to find the relative error of the sample average and standard deviation compared to the known values and compound them into an error metric for each pore and each sequence. The error metric is subsequently used for each pore to define a closeness metric, which will be minimized to predict the sequence. The accuracy is the ratio between the number of successful predictions and the total number of attempts. In (e) the orientation of the polymer is known and preserved when it passes through the different pores. In (f) the orientation of the polymer is not known and randomly changes when it passes through the different pores.

during multiple readouts of the unknown sequence, we need to consider all permutations of the two orientations in a given set of readouts. When we incorporate the possibility of different orientation in the translocation time measurements, then we would need to consider the sum of all possible permutations of orientations in determining the likelihood of the translocation times being part of a given distribution. This leads to $\mathcal{L}_{n}^{\text {ro }}=\left[\sum_{\text {perm }} \Pi_{i=1}^{m} \mathcal{P}_{n}^{\beta}\left(\tau_{i}\right)\right] \times$ $\left[\sum_{\text {perm }} \Pi_{i=1}^{m} \mathcal{P}_{n}^{\gamma}\left(\tau_{i}\right)\right] \cdots$ for the random orientation case. Again, we count the number of successful sequence detection events and plot the accuracy of this detection algorithm, as shown in Fig. 4(f). The result of this calculation shows that using the full distribution is surprisingly robust with respect to the randomization of the orientation, which is of paramount importance in practice. For the set of measurements which do not involve orientation effects we observe a distinctly faster detection of a sequence (95\% when there are 4 copies of each pore) as compared to the detection using the scatter plots [Fig. 4(e)]. With the orientations of the polymer as it enters the pore taken into account, the accuracy of detection rises to $95 \%$ with just 5 copies of each pore [Fig. 4(f)].

\section{CONCLUSION AND OUTLOOK}

In contrast to the generally accepted notion of suppressing the stochastic element of polynucleotide motion through nanopores to achieve efficient DNA sequencing, we propose to extract information from the statistical fluctuations towards sequence detection. Our strategy is based on designing distinguishable translocation time statistics for any given sequence by engineering the polymer-pore interactions and combining readouts from multiple pores for rapid convergence. The desired patterns in surface interaction could be achieved by using biological nanopores with appropriate modification $[55,56]$ or those with known hydrophobic-hydrophilic pattern structure [57], as well as solid-state nanopores with tailor-made surface interactions [15-19]. The proposed approach could potentially improve the overall speed of sequence detection by orders of magnitude, 
and could be integrated in high throughput microfluidic devices. The scope of our method is not limited by the specific patterning of the pore or the length of the polynucleotide and we now discuss some of the possible extensions.

Temporal modulations of the pore width can have a profound effect on translocation time distributions [58]. Such modulations could lead to significant gains in translocation times, and the features are particularly robust in the high-frequency regime. Incorporating such temporal oscillations in our model could enhance the distinguishing features of the translocation time distributions, resulting in better sequencing characteristics.

Another feature that would be extremely important in the study of DNA translocation, and one which we have not considered in our study, is the presence of secondary structures such as hairpins in the DNA. Imagine translocation through the differently patterned pores of a ssDNA punctuated with hairpin structures along its length [22]. The translocation process will be slowed down every time a secondary structure reaches the pore opening, requiring sufficiently strong driving force to break the hairpin bonds and allow ssDNA translocation. The translocation time distributions would differ depending on the number of bonds to be broken, which could lead to better distinguishability of ssDNA sequences using our statistical analysis.

A limitation to our approach in using patterned pores and statistical fluctuations to sequence DNA would be the complexity of pores and the consequent statistical analysis required for the sequencing of long chains of DNA with $1000 \mathrm{~s}$ of base pairs. However, long chains present problems in many sequencing techniques and are not specific to our method. A resolution to this problem using our approach would be to cut a large DNA into smaller segments which would be sent into parallel setups each of which has a series of pores. The current measurements of these individual segments as they pass each pore and subsequent analysis of the translocation time distributions should then reveal the sequence of the smaller segments and finally the sequence of the larger piece.

Although our method has been designed with DNA sequencing in mind, the proposed technique istransferablee to other biopolymer sensing needs. For example, a mixture of several biopolymer species could be rapidly sorted or counted. The polymer-pore specific interactions could then be tuned to the biopolymer hydrophobic/hydrophilic structure for discrimination.

\section{ACKNOWLEDGMENTS}

We would like to thank G. Battaglia for fruitful discussions. This work was supported by Grant No. EP/G062137/ 1 from the EPSRC.

\section{APPENDIX A: SENSITIVITY OF TRANSLOCATION TIME DISTRIBUTIONS ON PORE-POLYMER INTERACTION}

The translocation of a homopolymer through a narrow pore has been theoretically studied extensively in the past [28-51]. Luo et al. [29,32,33] investigated the porepolymer interaction in a uniformly attractive pore, which we chose as the control pore, Pore $\alpha$, in our simulations. In Fig. 5, we compare the potential landscapes for Pores $\alpha, \beta$, and $\gamma$, respectively. The potential landscapes seen by the polymer at the center of the pore along its length reveal the positions of the potential barriers for the three pores. Pore $\alpha$ has a strong barrier at the exit due to the stickiness of the pore. Pore $\beta$-which has a repulsive part in the middle-has a far lower barrier at the exit. It does,
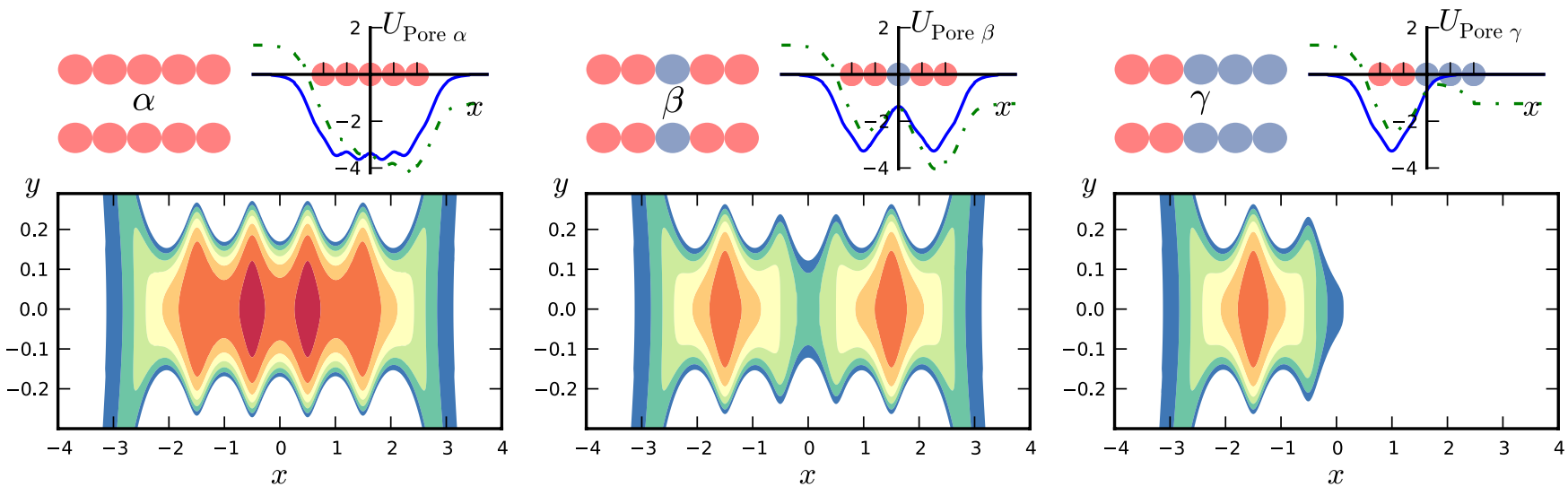

FIG. 5. Comparison of the polymer-pore interaction potentials. (Top left) A schematic of the pore. Pore monomers could either have an attractive (LJ) interaction (red) or a short-range repulsive (rLJ) interaction (blue) with the polymer inside the pore. (Top right) The potential energy landscape in the center $(y=0)$ along the length of the channel (blue) is modified (green) in the presence of an external driving force $F=0.5$. (Bottom) The complete potential energy landscape experienced by the polymer inside the pore. Blue to red represents increasing potential depth. 


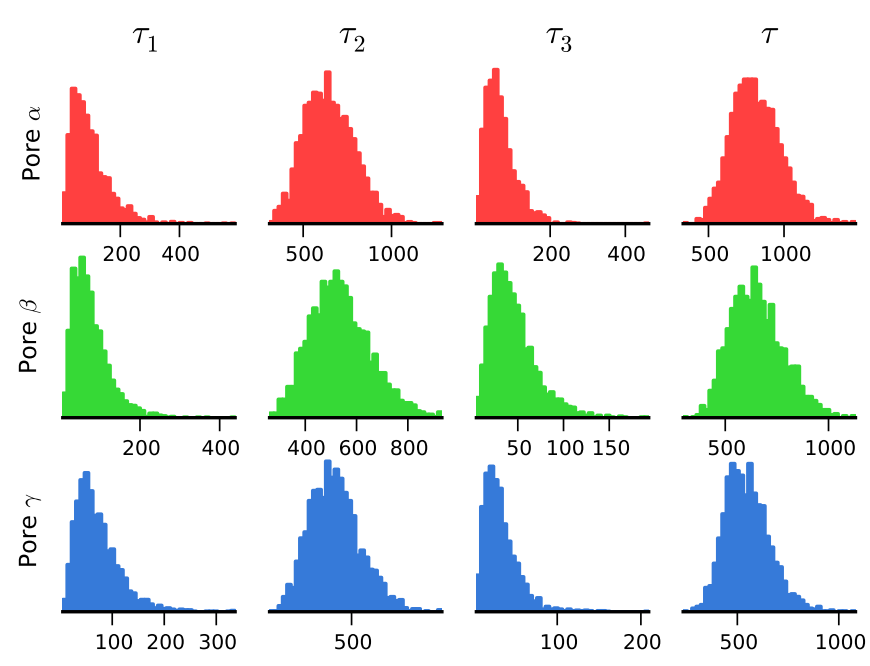

FIG. 6. Comparison of the filling, transfer, escape, and translocation time distributions for the three pores at $F=0.5$ and $\epsilon=1.0$.

however, experience a small barrier just after the entrance, which it overcomes easily. This explains why the filling time for Pore $\beta$ is lower than that of Pore $\alpha$. Pore $\gamma$, which has an attractive entrance but a repulsive exit, has a large barrier at the entrance, which makes the filling time relatively longer as compared with the other pores. However, the escape time for the polymer in Pore $\gamma$ is vastly reduced due to the repulsive exit.

In Figs. 6-8, we observe the change of the distribution for the translocation times with increasing attractive strengths, $\epsilon_{\mathrm{pm}}$ for the three different pores. For Pore $\alpha$, we note the transition from a Gaussian form to a longtailed distribution with increasing attraction, which was observed by Luo et al. This transition is observed in all the three pores, although for Pores $\beta$ and $\gamma$ they happen at higher attractive strengths.
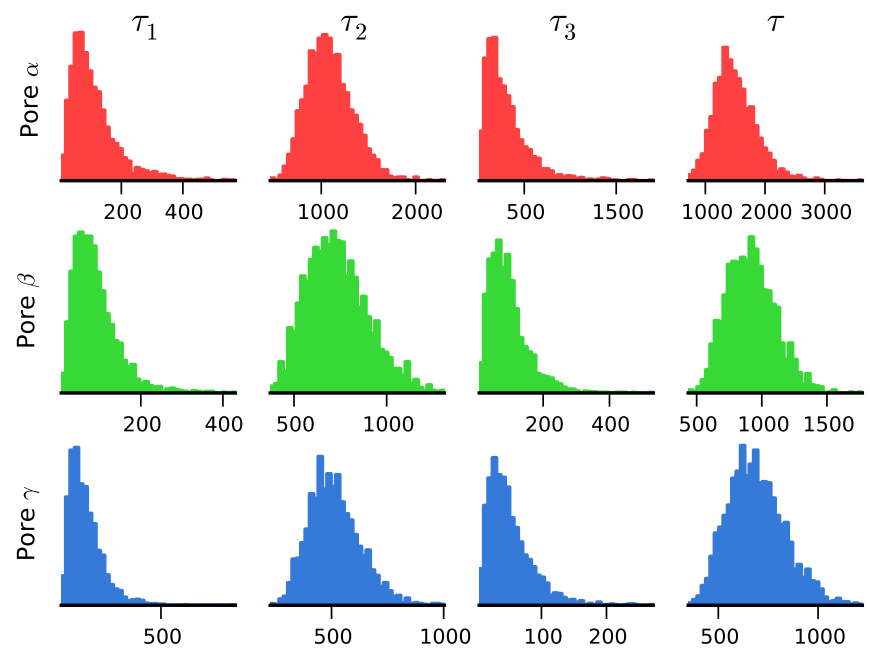

FIG. 7. Comparison of the filling, transfer, escape, and translocation time distributions for the three pores at $F=0.5$ and $\epsilon=1.5$.

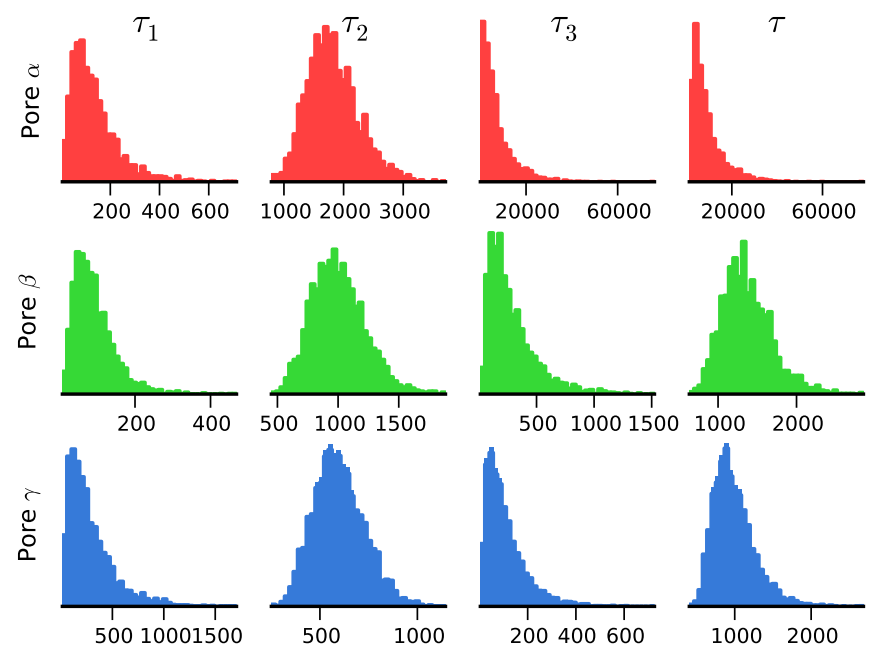

FIG. 8. Comparison of the filling, transfer, escape, and translocation time distributions for the three pores at $F=0.5$ and $\epsilon=2.0$.

\section{APPENDIX B: OPTIMIZING THE PORE FOR SEQUENCING: THE EFFECT OF DRIVING FORCE AND PORE WIDTH}

To understand the effects of patterning the pore on sequencing, we considered the translocation of heteropolymers through the pores. Following Luo et al. [34], the polymers were represented as consisting of symmetric blocks $A_{n} C_{n}$ of $\mathrm{A}$ and $\mathrm{C}$ bases, which interact differently with the pore. The time distributions for the three pores show a varying degree of sensitivity on the specific sequence of the polymer (Figs. 9-12), depending on the strength of the external force and the pore width. In Fig. 9, we show the dependence of the distributions for $F=1.0$ and $W=2.5$. For Pore $\alpha$, the difference in distributions for short block lengths is relatively small. As the

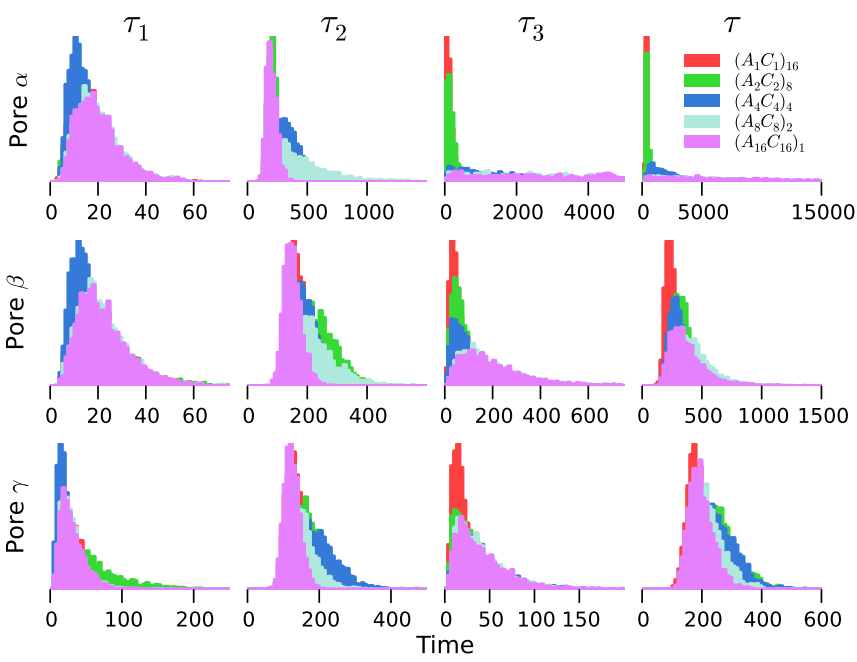

FIG. 9. Comparison of the filling, transfer, escape, and translocation time distributions for the three pores at $F=1.0$ and $W=2.5$ using heteropolymers with different sequences. 


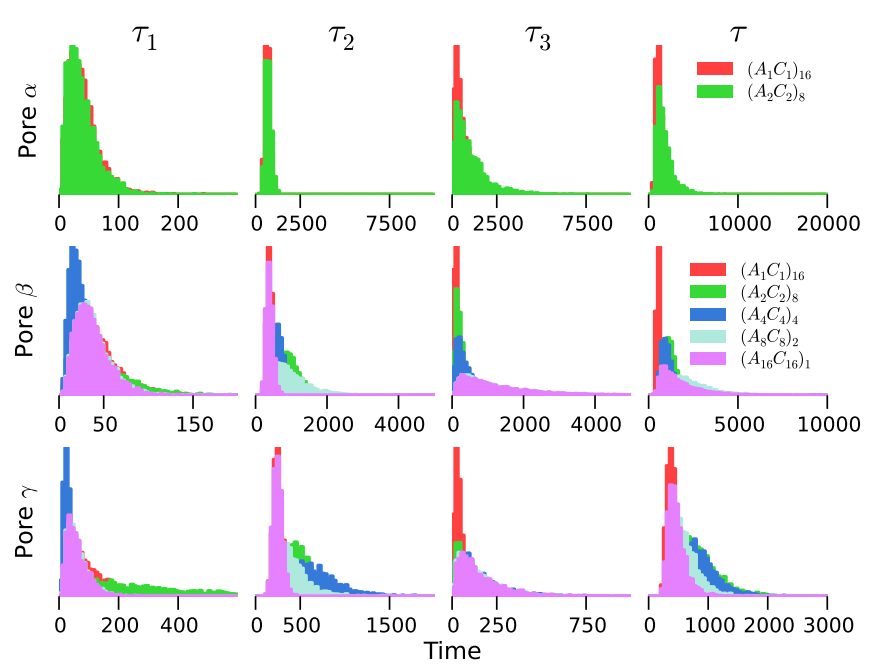

FIG. 10. Comparison of the filling, transfer, escape, and translocation time distributions for the three pores at $F=1.0$ and $W=2.25$ using heteropolymers with different sequences.

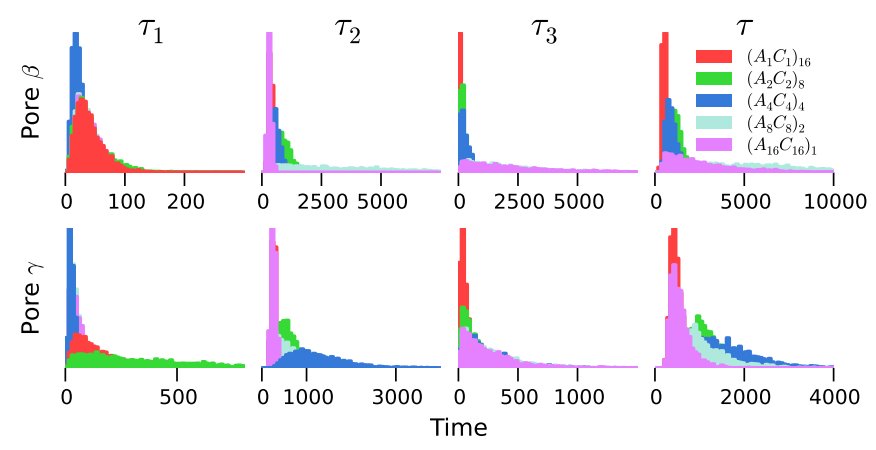

FIG. 11. Comparison of the filling, transfer, escape, and translocation time distributions for the Pores $\beta$ and $\gamma$ at $F=0.5$ and $W=2.5$ using heteropolymers with different sequences. Translocation through Pore $\alpha$ is extremely slow for this range of values and is not considered for this analysis.

block lengths are increased, the distribution changes sharply. However, for larger block lengths it becomes difficult again to distinguish them. For Pores $\beta$ and $\gamma$, the distributions have a high degree of overlap and are not suitable for sequencing.

As the width is decreased (Fig. 10, $W=2.25$ ), Pore $\alpha$ takes an extremely long time to translocate for larger block lengths. The potential barrier proves difficult to surmount, and the polymer is stuck for long periods inside the pore. However, lowering the width has a positive impact on Pore $\beta$, which leads to translocation time distributions that can be distinguished from one another. Although the translocation times are much longer, the distributions are well separated by their means and standard deviations. This impact is much less for Pore $\gamma$.

On the other hand, we could keep the pore width fixed $(W=2.5)$ and lower the strength of the external driving

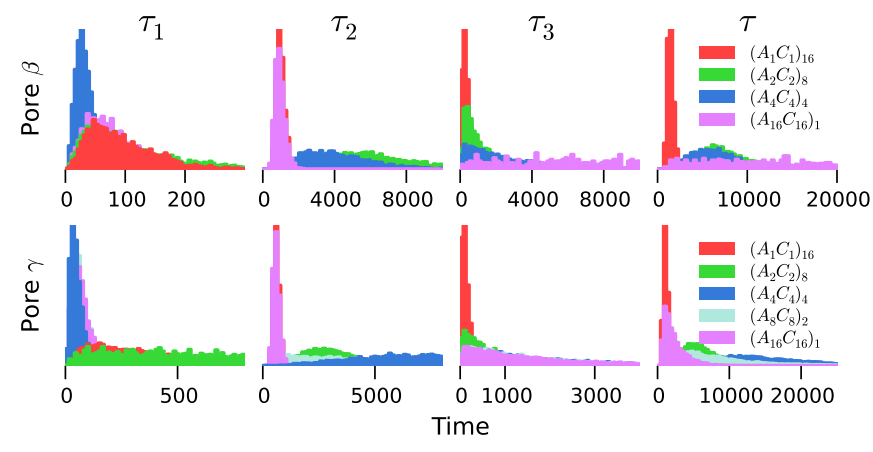

FIG. 12. Comparison of the filling, transfer, escape, and translocation time distributions for the Pores $\beta$ and $\gamma$ at $F=0.5$ and $W=2.25$ using heteropolymers with different sequences. Translocation through Pore $\alpha$ is extremely slow for this range of values and is not considered for this analysis.

force (Fig. 11). The effect on Pore $\alpha$ is drastic as the polymers fail to cross the potential barrier. Pore $\beta$ and $\gamma$, on the other hand, still translocate polymers although their distributions for the different sequences are far from distinguishable.

Finally, we keep the width at $W=2.25$ and lower the force to $F=0.5$ (Fig. 12). This completely takes out Pore $\alpha$ from consideration as the translocation times become prohibitively long. The translocation time scales are now much longer for Pores $\beta$ and $\gamma$ as well. However, the mean and standard deviations for Pore $\gamma$ are again well separated making it easier to distinguish between the distributions, and hence make it a suitable candidate for sequencing. In our simulations, we use Pore $\beta$ at $F=1.0, W=2.25$ and Pore $\gamma$ at $F=0.5, W=2.25$ as the two most suitable pores for our sequencer.

[1] R.F. Service, The Race for the $\$ 1000$ Genome, Science 311, 1544 (2006).

[2] J. Lagerqvist, M. Zwolak, and M. Di Ventra, Fast DNA Sequencing via Transverse Electronic Transport, Nano Lett. 6, 779 (2006).

[3] D. Branton et al., The Potential and Challenges of Nanopore Sequencing, Nat. Biotechnol. 26, 1146 (2008).

[4] J. Shendure and H. Ji, Next-Generation DNA Sequencing, Nat. Biotechnol. 26, 1135 (2008).

[5] J.A. Schloss, How to Get Genomes at One TenThousandth the Cost, Nat. Biotechnol. 26, 1113 (2008).

[6] M. Zwolak and M. Di Ventra, Colloquium: Physical Approaches to DNA Sequencing and Detection, Rev. Mod. Phys. 80, 141 (2008).

[7] S. K. Min, W. Y. Kim, Y. Cho, and K. S. Kim, Fast DNA Sequencing with a Graphene-based Nanochannel Device, Nature Nanotech. 6, 162 (2011).

[8] D.W. Deamer and D. Branton, Characterization of Nucleic Acids by Nanopore Analysis, Acc. Chem. Res. 35, 817 (2002).

[9] J. J. Kasianowicz, E. Brandin, D. Branton, and D.W. Deamer, Characterization of Individual Polynucleotide 
Molecules Using a Membrane Channel, Proc. Natl. Acad. Sci. U.S.A. 93, 13770 (1996).

[10] O. Braha et al., Designed Protein Pores as Components for Biosensors, Chem. Biol. 4, 497 (1997).

[11] M. Akeson, D. Branton, J. J. Kasianowicz, E. Brandin, and D. W. Deamer, Microsecond Time-Scale Discrimination among Polycytidylic Acid, Polyadenylic Acid, and Polyuridylic Acid as Homopolymers or as Segments within Single RNA Molecules, Biophys. J. 77, 3227 (1999).

[12] A. Meller, L. Nivon, E. Brandin, J. Golovchenko, and D. Branton, Rapid Nanopore Discrimination Between Single Oligonucleotide Molecules, Proc. Natl. Acad. Sci. U.S.A. 97, 1079 (2000).

[13] A. Meller, L. Nivon, and D. Branton, Voltage-Driven DNA Translocations through a Nanopore, Phys. Rev. Lett. 86, 3435 (2001).

[14] A. Meller, Dynamics of Polynucleotide Transport through Nanometer-Scale Pores, J. Phys. Condens. Matter 15, R581 (2003).

[15] A. J. Storm, J. H. Chen, X. S. Ling, H. W. Zandbergen, and C. Dekker, Fabrication of Solid-State Nanopores with Single-Nanometre Precision, Nature Mater. 2, 537 (2003).

[16] M. J. Kim, M. Wanunu, D. C. Bell, and A. Meller, Rapid Fabrication of Uniformly Sized Nanopores and Nanopore Arrays for Parallel DNA Analysis, Adv. Mater. 18, 3149 (2006).

[17] T. Ohshiro and Y. Umezawa, Complementary Base-PairFacilitated Electron Tunneling for Electrically Pinpointing Complementary Nucleobases, Proc. Natl. Acad. Sci. U.S.A. 103, 10 (2006).

[18] S. M. Iqbal, D. Akin, and R. Bashir, Solid-State Nanopore Channels with DNA Selectivity, Nature Nanotech. 2, 243 (2007).

[19] M. Wanunu and A. Meller, Chemically-Modified SolidState Nanopores, Nano Lett. 7, 1580 (2007).

[20] P. Chen et al., Atomic Layer Deposition to Fine-tune the Surface Properties and Diameters of Fabricated Nanopores, Nano Lett. 4, 1333 (2004).

[21] V. Tabard-Cossa, D. Trivedi, M. Wiggin, N. N. Jetha, and A. Marziali, Noise Analysis and Reduction in Solid-State Nanopores, Nanotechnology 18, 305505 (2007).

[22] I. M. Derrington, T.Z. Butler, M. D. Collins, E. Manrao, M. Pavlenok, M. Niederweis, and J.H. Gundlach, Nanopore DNA Sequencing with MspA, Proc. Natl. Acad. Sci. U.S.A. 107, 16060 (2010).

[23] J. Clarke, H. Wu, L. Jayasinghe, A. Patel, S. Reid, and H. Bayley, Continuous Base Identification for SingleMolecule Nanopore DNA Sequencing, Nature Nanotech. 4, 265 (2009).

[24] D. Stoddart, A. J. Heron, E. Mikhailova, G. Maglia, and H. Bayley, Single-Nucleotide Discrimination in Immobilized DNA Oligonucleotides with a Biological Nanopore, Proc. Natl. Acad. Sci. U.S.A. 106, 7702 (2009).

[25] S. Polonsky, S. Rossnagel, and G. Stolovitzky, Nanopore in Metaldieletric Sandwich for DNA Position Control, Appl. Phys. Lett. 91, 153103 (2007).

[26] A. R. Hall, A. Scott, D. Rotem, K. K. Mehta, H. Bayley, and C. Dekker, Hybrid Pore Formation by Directed Insertion of $\alpha$-haemolysin into Solid-State Nanopores, Nature Nanotech. 5, 874 (2010).
[27] E. E. Schadt, S. Turner, and A. Kasarskis, A Window into Third-Generation Sequencing, Human Molecular Genetics 19, R227 (2010).

[28] D. K. Lubensky and D. R. Nelson, Driven Polymer Translocation through a Narrow Pore, Biophys. J. 77, 1824 (1999).

[29] I. Huopaniemi, K. Luo, T. Ala-Nissila, and S. C. Ying, Langevin Dynamics Simulations of Polymer Translocation through Nanopores, J. Chem. Phys. 125, 124901 (2006).

[30] M. Muthukumar and C. Y. Kong, Simulation of Polymer Translocation through Protein Channels, Proc. Natl. Acad. Sci. U.S.A. 103, 5273 (2006).

[31] S. Matysiak, A. Montesi, M. Pasquali, A. B. Kolomeisky, and C. Clementi, Dynamics of Polymer Translocation through Nanopores. Theory Meets Experiment, Phys. Rev. Lett. 96, 118103 (2006).

[32] K. Luo, T. Ala-Nissila, S. C. Ying, and A. Bhattacharya, Influence of Polymer-Pore Interactions on Translocation, Phys. Rev. Lett. 99, 148102 (2007).

[33] K. Luo, T. Ala-Nissila, S. C. Ying, and A. Bhattacharya, Dynamics of DNA Translocation through an Attractive Nanopore, Phys. Rev. E 78, 061918 (2008).

[34] K. Luo, T. Ala-Nissila, S. C. Ying, and A. Bhattacharya, Sequence Dependence of DNA Translocation through a Nanopore, Phys. Rev. Lett. 100, 058101 (2008).

[35] M. G. Gauthier and G. W. Slater, Sequence Effects on the Forced Translocation of Heteropolymers through a Small Channel, J. Chem. Phys. 128, 175103 (2008).

[36] B. Luan et al., Base-by-Base Ratcheting of Single Stranded DNA through a Solid-State Nanopore, Phys. Rev. Lett. 104, 238103 (2010).

[37] A. Nikoubashman and C. N. Likos, Flow-Induced Polymer Translocation through Narrow and Patterned Channels, J. Chem. Phys. 133, 074901 (2010).

[38] W. Sung and P. J. Park, Polymer Translocation through a Pore in a Membrane, Phys. Rev. Lett. 77, 783 (1996).

[39] M. Muthukumar, Polymer Translocation through a Hole, J. Chem. Phys. 111, 10371 (1999).

[40] M. Muthukumar, Translocation of a Confined Polymer through a Hole, Phys. Rev. Lett. 86, 3188 (2001).

[41] J. Chuang, Y. Kantor, and M. Kardar, Anomalous Dynamics of Translocation, Phys. Rev. E 65, 011802 (2001).

[42] M. Muthukumar, Polymer Escape through a Nanopore, J. Chem. Phys. 118, 5174 (2003).

[43] R. Metzler and J. Klafter, When Translocation Dynamics becomes Anomalous, Biophys. J. 85, 2776 (2003).

[44] E. Slonkina and A. B. Kolomeisky, Polymer Translocation through a Long Nanopore, J. Chem. Phys. 118, 7112 (2003).

[45] Y. Kantor and M. Kardar, Anomalous Dynamics of Forced Translocation, Phys. Rev. E 69, 021806 (2004).

[46] A. Milchev, K. Binder, and A. Bhattacharya, Polymer Translocation through a Nanopore Induced by Adsorption: Monte Carlo Simulation of a CoarseGrained Model, J. Chem. Phys. 121, 6042 (2004).

[47] U. Gerland, R. Bundschuh, and T. Hwa, Translocation of Structured Polynucleotides through Nanopores, Phys. Biol. 1, 19 (2004).

[48] A. Gopinathan and Y. W. Kim, Polymer Translocation in Crowded Environments, Phys. Rev. Lett. 99, 228106 (2007). 
[49] C.T.A. Wong and M. Muthukumar, Polymer Translocation through $\alpha$-hemolysin Pore with Tunable Polymer-Pore Electrostatic Interaction, J. Chem. Phys. 133, 045101 (2010).

[50] A. Milchev, Single-Polymer Dynamics under Constraints: Scaling Theory and Computer Experiment, J. Phys. Condens. Matter 23, 103101 (2011).

[51] R.H. Abdolvahab, M. R. Ejtehadi, and R. Metzler, Sequence-Dependence of the Binding Energy in Chaperone-driven Polymer Translocation through a Nanopore, Phys. Rev. E 83, 011902 (2011).

[52] M.P. Allen and D. J. Tildesley, Computer Simulation of Liquids (Oxford University, New York, 1987).

[53] A. F. Sauer-Budge, J. A. Nyamwanda, D. K. Lubensky, and D. Branton, Unzipping Kinetics of Double-Stranded DNA in a Nanopore, Phys. Rev. Lett. 90, 2381011 (2003).
[54] U. F. Keyser et al., Direct Force Measurements on DNA in a Solid-State Nanopore, Nature Phys. 2, 473 (2006).

[55] W. W. Li, T. D. W. Claridge, Q. Li, M. R. Wormald, B. G. Davis, and H. Bayley, Tuning the Cavity of Cyclodextrins: Altered Sugar Adaptors in Protein Pores, J. Am. Chem. Soc. 133, 1987 (2011).

[56] A. F. Hammerstein, L. Jayasinghe, and H. Bayley, Subunit Dimers of $\alpha$-hemolysin Expand the Engineering Toolbox for Protein Nanopores, J. Biol. Chem. 286, 14324 (2011).

[57] M. Mahfoud, S. Sukumaran, P. Hülsmann, K. Grieger, and M. Niederweis, Topology of the Porin MspA in the Outer Membrane of Mycobacterium Smegmatis, J. Biol. Chem. 281, 5908 (2005).

[58] J. A. Cohen, A. Chaudhuri, and R. Golestanian, Active Polymer Translocation through Flickering Pores, Phys. Rev. Lett. 107, 238102 (2011). 